\title{
Immunopathology of nerve damage in leprosy
}

\author{
M ELLIOTT \\ The London Hospital Medical College, (Whitechapel), Turner \\ Street, London E1 $1 B B$
}

Received for publication 20 October 1980

Editorial Note. For nearly 10 years, first in Oxford, later in Birmingham and Edinburgh, and later still for all medical schools in the United Kingdom, the British Leprosy Relief Association (LEPRA) has sponsored prize essay competitions. These have recently been extended to Zambia and India. We present here an abbreviated version of a prize-winning essay in 1979, from a student in one of the London Hospitals. Following a general introduction to the immunopathology of leprosy, including sections dealing with the nature of the immunological defect and the response of the immune system to Mycobacterium leprae, the author proceeds as follows.

\section{Nerve damage in leprosy}

Often many of the clinical features of a disease are due to local tissue damage as a result of the host's attempts to eliminate the organism. Mycobacterium leprae requires CMI for its elimination and this can also cause extensive tissue damage leading to the clinical manifestations of the disease. Interaction between humoral antibody and antigen derived from the organism can cause tissue damage even though the antibody is unable to eliminate the organism.

Nerve damage in leprosy is recognized more easily histologically than functionally; since, due to receptive field overlap, $30 \%$ of the fibres in a nerve must be destroyed before decreased sensation in the area subserved can be recognized. Damage occurs more readily in tuberculoid leprosy than lepromatous leprosy. In the former it is a feature early on whereas in the latter damage and partial loss of sensation is a feature of the advanced disease. It occurs either as part of the natural history of the disease or due to reactions.

The peripheral nerves are something of an immunological backwater. The perineurium forms an effective barrier and the endothelial cells of the intraneural capillaries have tight junctions and hence are less permeable than elsewhere in the body. The Schwann cells, which protect and support the axon, have a very long life span and anything which is taken into their cytoplasm may not be exposed to the extracellular environment for years. Soluble antigens may leak out but even so infection in this sheltered zone can escape recognition. Extraneural bacilli are phagocytosed by histiocytes, which have a life span of a few weeks. Again soluble antigens are exposed all the time but when the host cell dies the whole bacilli will be exposed.

$M$. leprae is the only organism known to parasitize Schwann cells and it is important to understand how it gets there. Possible mechanisms are:

0305-7518/81/052355 + $07 \$ 01.00$ @ British Leprosy Relief Association 
(1) Phagocytosis by Schwann cells in the upper layers of the dermis and once engulfed the bacteria travel centrally through contiguous Schwann cells or possibly within the axon itself. However there is little evidence that bacilli ever lie free in the dermis and histologically nerves in the middle and deeper layers of the dermis are involved to a greater extent than terminal nerve fibres. Bacilli are seldom seen in axons and when they are seen there the possibility that they are not in the tongues of Schwann-cell cytoplasm invaginating the axon has not been excluded. ${ }^{1}$

(2) By penetration of the perineurium with the inflammatory cells. However when the inflammatory cells enter, the Schwann cells are already laden with bacilli and hence bacillation is a cause rather than a consequence of inflammatory cell entry.

(3) Via the endoneural blood vessels. Bacteraemia is a normal finding in untreated lepromatous patients and histologically, in early nerve involvement, bacilli tend to be close to the endoneural blood vessels.

Much is still unknown, for instance whether the bacteria enter the nerve within macrophages or free, however once inside ultimate phagocytosis by Schwann cells is inevitable.

The predilection of $M$. leprae for nerves is as yet not satisfactorily explained. Other mycobacteria are not seen to enter nerves. It is possible that it represents an immunological phenomenon in that elsewhere in the body $M$. leprae is destroyed whereas since the nerve is an immunological backwater it is able to proliferate there. In lepromatous patients, in whom the immune response to $M$. leprae is generally depressed, bacteraemia is a normal finding and the visceral organs are often involved with granuloma formation. Studies of liver and kidney biopsies from LL patients show that the bacteria are grossly fragmented as opposed to the intact bacteria seen in skin and nerves. ${ }^{2}$

Other theories are that the peripheral nerves and skin are cooler and it is possible that $M$. leprae multiplies more rapidly here or that these sites are subjected to constant minor trauma leading to minor inflammation with consequent increased capillary stickiness and bacillary or monocyte adherence. If the latter is true it is at variance with the hypothesis that the nerve is an immunological backwater.

In the initial stages of the infection Schwann cells are colonized but little affected. However after some time they may be destroyed by either the bulk of organisms or competition with metabolic processes. Job and Verghese ${ }^{3}$ found increased numbers of mitochondria, ribosomes and increased endoplasmic reticulum in infected cells. With cell destruction the bacilli are released and may be taken up by neighbouring Schwann cells, hence moving along the nerve, or by intraneural histiocytes, which could transport them via endoneural blood vessels along the nerve. In tuberculoid leprosy the bacilli are far more concentrated in the nerves than elsewhere and this is probably the major route of dissemination in this form of the disease.

The mechanism of tissue damage is different in the different forms of the disease and this will now be discussed.

(1) Tuberculoid leprosy. In this form of the disease CMI against M. leprae is high and the evidence that this is responsible for tissue damage and the clinical presentation is:

(a) Histological.

(b) The intensity of inflammatory changes is correlated with the strength of delayed hypersensitivity reactions as measured by LTT and LMIT responses to M. leprae. Clinical severity of the disease is also related to the spread of $M$. leprae.

(c) Mice made lepromatous develop tuberculoid-type lesions with nerve damage when injected with syngeneic lymphoid cells or thymus transplants.

(d) The localized nature of the lesions makes it unlikely that they are caused by autoimmune responses precipitated by $M$. leprae infection. However involvement of responses to host cell antigens altered by $M$. leprae parasitism cannot be excluded. 
The precise nature of tissue damage is uncertain; for instance the exact role of $T$ cell cytotoxicity or cytotoxic mediators remains unknown. Mere infiltration and distortion of the tissues by the inflammatory cells with intra and extracellular oedema may explain most of the features of the lesion. Its chronic nature is probably related to the extremely slow degradation and elimination of $M$. leprae.

The overall picture is of a wave of bacillation spreading slowly through dermal nerves pursued but never quite overtaken by the inflammatory response. Once all the nerve is destroyed the bacilli are finally destroyed with it and resolution by fibrosis occurs leading to the clear centre of the tuberculous lesion.

(2) Lepromatous leprosy. In this form of the disease CMI directed against M. leprae is depressed and some other mechanism of nerve damage must be sought.

Hastings ${ }^{4}$ states that the concentration of bacilli in perineural cells is as great as or more than that in Schwann cells and that both sites may be damaged but that perineural damage is the more important. The perineurium plays an important part in stabilizing the intraneural environment, so important for the normal function of the nerve. Bacillary multiplication is thought to initiate perineural breakdown, by some unknown mechanism, leading to perineural incompetence. Because of this ECF can enter the nerve; impairment will be reversible which would account for the improvement in lepromatous leprosy upon treatment. Similarly the nerve is more vulnerable to the entry of inflammatory cells. The histological picture does not support either of these views. Lepromatous lesions are characterized by the lack of inflammatory cells and there are structural alterations in the nerve which do not concur with the view that impairment of nerve activity is purely functional due to alterations in the extraneural environment. If this were true it would be expected that all the axons in the nerve trunk would be similarily affected since they share the same extra axonal environment. This is not so.

Alternatively the structural abnormalities may be due to defective attempts at repairing the damaged perineurium. Experimentally Morris et $a l^{5}$ found that Schwann cells are involved in perineural repair by putting out pseudopodia, which plug gaps in the perineurium, forming a pseudoperineurium, which becomes organized and indistinguished from the normal. $\mathrm{Job}^{6}$ found in electronmicrograph studies of radial cutaneous nerve biopsies from 5 untreated LL patients that Schwann cells are hypertrophied and have papillary processes resembling pseudopodia. In the intraneural blood vessels endothelial swelling and hypertrophy leading to narrowed or obstructed lumina was seen. In some cases there was proliferation of perineural cells forming several layers and the number of myelinated nerve fibres was decreased. In others, axons surrounded by bacilli laden Schwann cells looked normal; in between these extremes there was a graduation of degenerative change.

Schwann cells, perineural cells, endothelial cells and macrophages were all bacillated and showed foamy degeneration. Schwann cell bacillation may lead to defective patching of perineural damage and hence it continues unchecked leading to the multilayered perineural appearance.

Loss of function in the axons is probably due to loss of function in the supporting Schwann cells because of bacillary competition for metabolites etc. This will be exacerbated by blood vessel obstruction and perineural incompetence. Nerve damage in lepromatous leprosy is therefore a secondary phenomenon.

(3) Reversal reactions. The clinical presentation varies widely but it can be divided basically into 3 groups:

(a) Those with predominantly skin hypersensitivity.

(b) Those with predominantly nerve hypersensitivity.

(c) Those with both.

Barnetson $e t a l^{7}$ investigated the mechanisms underlying these 3 groups. In all reactions 
it was found that there was an increase in LTT but that nerve and skin involvement are associated with different antigens. Whole washed bacilli failed to elicit high responses in nerve reactions suggesting that cytoplasmic antigens play an important role in this condition, whereas in the skin case, the increased response is chiefly directed at whole washed bacilli and therefore surface antigens are more important. In BT, BB and BL patients, who are reasonably immunologically competent, many bacilli may be present in the nerve but fewer are present in the skin. Cell surface antigens are rarely exposed and soluble cytoplasmic antigens are more likely to be exposed but are released slowly. In reactions there is a sudden increase in release, due to an unknown trigger mechanism, with subsequent destruction of Schwann cells and exposure of bacillary surface antigens. In the skin case LTT results indicated that there may be an increased exposure of surface antigens. In reactions there is a rise in $\operatorname{IgG}, \operatorname{IgM}$ and IgA levels but this is probably a non-specific effect rather than a cause.

Godal et $a l .{ }^{8}$ studied reversal reactions in $10 \mathrm{BT}$ patients and found that although they showed clinical deterioration, this coincided with a very strong immune response to $M$. leprae. That the reaction is due to an increase in CMI has been confirmed in experiments by Gaugas et al. ${ }^{9}$ in which implantation of isogeneic thymus tissue caused changes in infected mice similar to those seen in humans.

Also Rees and Wedell ${ }^{10}$ took thymectomized, irradiated mice and injected them intraperitoneally with lymphocytes equivalent to those present in the normal mouse 10 months after the injection of $M$. leprae, when macroscopic lesions had already developed in the foot pad. Six to 10 days later, the areas of skin in which $M$. leprae were present became red and swollen. Bacteriological examination showed that the bacilli degenerate and the yield of organisms drops enormously. Histologically there was evidence of increased lymphocytic infiltration. This is similar to what is seen in the lepromatous patient undergoing a reversal reaction.

Factors precipitating reversal reactions remain uncertain though antileprosy drugs and BCG have been implicated; whatever they are the primary feature is increased CMI against $M$. leprae with oedema which is responsible for the early stages of the lesions. Further epithelioid cell formation leads to total tissue destruction.

Reversal reactions represent a serious therapeutic problem. Immunosuppressive drugs may be necessary to control them and prevent extensive nerve damage. Attempts to increase CMI to eliminate the organisms should be carried out very carefully indeed since they may precipitate a reaction.

(4) ENL. The nerves are painful and tender for prolonged periods and still show only mild loss of function. The pain is probably chemically induced by release of active substances, e.g. polypeptides from damaged cells in the vicinity of the nerves.

The lesions occur in areas of high bacillary concentration, i.e. perineural cells, and thus surviving Schwann cells are less liable to be damaged. This explains the unexpectedly slight damage caused. However ${\mathrm{J} o b^{6}}^{6}$ says that bacilli are less common in perineural cells than Schwann cells.

ENL is not associated with a shift in cellular immune status and is probably associated with humoral immune mechanisms. There is a decrease in the number of bacilli, which undergo considerable degeneration undoubtedly with release of antigenic material into the circulation.

However direct evidence that ENL is caused by immune complexes is still lacking and it must be explained why one third of patients with evidence of circulating complexes do not develop ENL. Also why do most patients have only a few attacks while others have repeated attacks over a long period? It could be due to variations in the amount of bacilli being killed off by residual CMI, determined by varying host/parasite relationships. 


\section{Conclusions}

It can be seen that the understanding of the immunopathology of leprosy is far from clear. In an attempt to bring all the evidence and arguments together I will postulate a hypothesis, which seems to fit the evidence. Any theory must explain several important features of the disease:

(a) how the infection becomes established and what determines the subsequent course of the disease;

(b) why the bacteria shows a predilection for nerves and skin;

(c) how tissue damage is brought about?

In the race between the immune system's attempt at elimination and bacterial proliferation the crucial question is who wins and by how much? Due to the complexity and enormous variations in the disease it is doubtful that any one factor will determine this. It is likely that a number of factors make different contributions in different cases.

To establish an infection in experimental mice it was necessary to immunosuppress them which suggests that normally the immune system is able to cope with infection. Specific and non-specific factors are important and anything which decreases the effectiveness of either of these could prove to be important in the establishment and subsequent course of the disease. Non-specific factors include: diet, climate, concurrent infection, general socioeconomic conditions, age, sex, genetic factors, etc.

On the specific side tolerance has been mentioned and it is possible that the frequency, route and inoculum size of infecting organisms as well as the state of the host immune system are very important. For a long time it was thought that prolonged exposure was necessary for infection to occur however this is not compatible with the finding of explosive spread of leprosy when introduced into non-endemic areas. From the study by Godal and Negassi ${ }^{11}$ it appears that subclinical infection is the most common outcome of exposure to $M$. leprae and that supraexposure may lead to depression of the host response and establishment of the infection.

In terms of antigen predosage there is high and low zone tolerance. Between these there is stimulation of active immunity. The spectrum is continuous, hence interaction between the immune system and the organism will give a response lying somewhere on this curve and its position will determine subsequent events. Sensitization will result in subclinical infection. Tolerance, depending upon its degree, will lead to clinical infection; where tolerance nearly equals sensitization reactivity is more likely to be regained and hence the disease will be towards the tuberculoid pole. When tolerance is much more firmly established lepromatous leprosy will be the outcome. Tolerance depends upon the continued presence of antigen for its maintainance and it may be that in tuberculoid leprosy $M$. leprae gets hidden away inside nerves and fails to render newly produced lymphocytes tolerant. This primary response would explain the slow time course of damage in tuberculoid leprosy. In reactions, damage occurs much more rapidly by the same mechanism and this could represent a change to 'sensitization'.

The pattern of exposure to the immune system could be determined by non-specific immune (e.g. BCG) and non-immune (e.g. drugs) factors and hence varied by changes in these leading to shifts on the leprosy spectrum.

The inability of lepromatous macrophages to lyse M. leprae is a property of live bacilli and suggests that it is resistant to lysis, however the experiments of Convit et al., ${ }^{12}$ in which lepromatous macrophages could lyse $M$. leprae when other mycobacteria were administered concurrently, suggests that the macrophage itself is in some way deficient. In granulomata in other tissues the bacteria are fragmented whereas those in nerves are whole. M. leprae 
is known to be very fastidious in its growth requirements and it may be that Schwann cells are its first choice home but the organism can survive in other tissues, but considerably less well. By this mechanism bacterial proliferation is decreased in extraneural tissue and the immune response is here able to tip the balance in favour of elimination; this explains the fragmentation of bacilli in liver and kidney granulomata seen in LL leprosy. Similarly, lysis of dead but not live bacteria, intraneurally, is explained in this way.

The activation of macrophages by administration of other mycobacterial antigens probably represents a non-specific affect tipping the balance in favour of elimination. The picture is further complicated by the thesis that the nerve is an immunological backwater. The immune response may be sufficient to eliminate bacteria elsewhere but is unable to completely overcome the barriers where the nerve is involved. In lepromatous leprosy the immune response is not even sufficient to eliminate extraneural bacteria. Reversal reactions represent a situation where the balance is tipped strongly in favour of elimination and massive cell-mediated destruction of tissue occurs wherever bacteria are exposed as opposed to the gradual destruction in tuberculoid leprosy where the difference between elimination and proliferation is much less.

Whatever the mechanism of predilection the bacilli become established in the Schwann cells. In lepromatous leprosy they proliferate rapidly and competition for metabolites etc. leads to decreased Schwann-cell function with consequent axon demyelination and, due to inefficient repair of the damaged perineurium, the characteristic histological picture of lepromatous nerves. Decreased Schwann-cell function may lead to defective antigen presentation to $\mathrm{T}$ cells making things even worse. The $\mathrm{T}$ cells and macrophages probably affect each other reciprocally. Decreased $\mathrm{T}$ cell function affecting the macrophage adversely and vice versa. In tuberculoid leprosy the bacilli are much fewer and the host in attempting to destroy the bacilli destroys the Schwann cells with secondary demyelination. However bacillary multiplication outstrips this and the picture is one of a wave of bacillation followed down the nerve by a wave of tissue destruction.

The time after infection that the bacteria enters the nerve is very difficult to ascertain because of the long incubation period of the disease, which is commonly believed to be in the order of $2-5$ years. In view of all that has been said it is likely that its length is determined by the relative excess of proliferation over elimination. It is likely, if the endoneural environment is the most favourable for growth and if the infection is to be established, that $M$. leprae colonizes these cells very early on. Subclinical infection represents the situation where the host is sensitized and M. leprae is eliminated before it is able to reach the Schwann cells.

Initially the presence or absence of lymphocyte tolerance to $M$. leprae will determine whether the bacteria reaches the Schwann cells, and indeed other cells of the body, but if it becomes established here this further contributes to the immunological defect by decreased macrophage function.

Leprosy can therefore be seen to be a disease in which the immune system plays a major part in the disease process.

\section{References}

${ }^{1}$ Pearson JMH, Ross WF. Nerve involvement in leprosy. Pathology differential diagnosis and principles of management. Lepr Rev, 1975; 46: 199-212.

2 Dratz D, Chen DT, Weng Hziang Lu. The continuous bacteraemia of lepromatous leprosy. $N$ Engl J Med, 1972; 287: 159.

${ }^{3}$ Job CK, Verghese R. Schwann cell clfanges in lepromatous leprosy-an electronmicroscope study. Indian J Med Res, 1975; 63: 897-901. 
${ }^{4}$ Hastings RL. Transfer Factor as a probe of the immune defect in lepromatous leprosy. Int J Lepr. 1977; 45: 281-91.

${ }^{5}$ Morris JH, Hudson AR, Weddell AGM. A study of degeneration and regeneration in the divided rat sciatic nerve based on electron-microscopy. Z Zellforsch mickrosk Anat, 1972; 124: 165.

${ }^{6}$ Job CK. M. leprae in nerve lesions in lepromatous leprosy. Arch Path, 1970; 89: 195208.

${ }^{7}$ Barnetson RS et al. Cell mediated and humoral immunity in 'reversal reactions'. Int J Lepr, 1976; 44: 267-74.

${ }^{8}$ Godal T, Myrvang B, Samuel DR, Ross WF, Löfgren M. Mechanism of reactions in borderline tuberculoid leprosy. Acta Path et Microbiol Scand Sect A, suppl, 1973; 236: $45-53$.

${ }^{9}$ Gaugas JM, Rees RJW, Weddell AGM, Palmer E. Reversal effects of thymus grafts on lepromatous leprosy in thymectomized irradiated mice. Int J Lepr, 1971; 39: 388-95.

10 Rees RJW, Weddell AGM. Experimental models for studying leprosy. Ann NY Acad Sci, 1968; 154: 214-36.

11 Godal T, Negassi K. Subclinical infection in leprosy. BMJ, 1973; 3: 557-9.

12 Convit J, Avila JL, Goilaman M. et al. Elimination of M. leprae subsequent to local in vivo activation of macrophages in lepromatous leprosy by other mycobacteria. Clin Exp Med, 1974; 17: 261-5. 